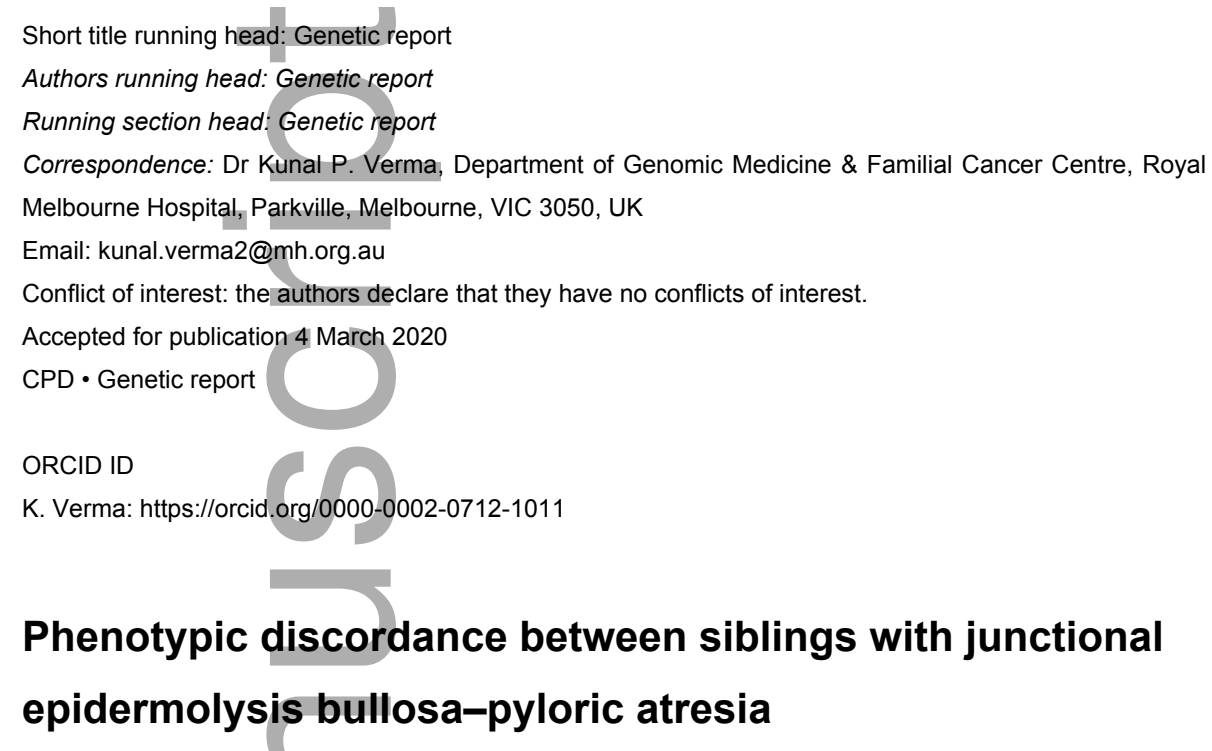

\section{Phenotypic discordance between siblings with junctional epidermolysis bullosa-pyloric atresia}

K. P. Verma, ${ }^{1}$ S. J. Robertson ${ }^{2}$ and I. M. Winship ${ }^{1,3}$

${ }^{1}$ Department of Genomic Medicine and Familial Cancer Centre and ${ }^{1}$ Department of Dermatology, Royal Melbourne Hospital,
Melbourne, Australia; and ${ }^{2}$ Department of Medicine, University of Melbourne, Melbourne, Australia

Junctional epidermolysis bullosa with pyloric atresia (JEB-PA) is an autosomal recessive disease characterized by epithelial fragility and PA. Cleavage occurs within the lamina lucida of the skin basement membrane zone. Biallelic pathogenic variants in ITGA6 and ITGB4 are responsible, the latter being more common. ${ }^{1,2}$ Loss of skin and mucous membrane barrier and absorptive function results in malnutrition and risk of sepsis. Other extracutaneous manifestations include dental caries, genitourinary abnormalities and rudimentary ears ${ }^{1}$. Prognosis is usually poor, with reduced life expectancy predicted in most cases. ${ }^{2}$ Two notable EB subtypes that share similarities with JEB-PA are EB simplex with PA (EBS-PA) due to PLEC variants, and JEB with respiratory and renal involvement (JEB-RR) caused by ITGA3 variants. ${ }^{1}$

Our proband, a 36-year-old Lebanese man, had skin blistering and pyloric stenosis at birth; the stenosis was surgically corrected at 3 days of age. His parents were consanguineous. The patient's older sister had died 5 years previously at the age of 14 months, having had skin blistering, severe diarrhoea, protein-losing enteropathy (PLE), failure to thrive (FTT) and sepsis; histology of her bowel mucosa revealed epithelial clefting at the lamina propria. Postmortem examination revealed extensive de-epithelialization of the skin and small intestine, without PA.

The proband's neonatal skin biopsy revealed epidermal separation at the lamina propria. In childhood, he experienced diarrhoea, FTT and continued skin blistering. Aged 7 years, he deteriorated, developing PLE and requiring parenteral nutrition and albumin replacement. Intermittent stridor was noted. Endoscopy This is the author manuscript accepted for publication and has undergone full peer review but has not been through the copyediting, typesetting, pagination and proofreading process, which may lead to differences between this version and the Version of Record. Please cite this article as doi: $10.1111 /$ CED.14223

This article is protected by copyright. All rights reserved 
was planned but the patient could not be endotracheally intubated owing to supraglottic stenosis. Subsequent colonic mucosal biopsy showed separation of the mucosa from the lamina propria; electron microscopy of repeat skin biopsies showed small numbers of poorly formed hemidesmosomes and wellformed anchoring fibrils. The diagnosis of JEB was made.

Aged 8 years, the patient developed urethral stenosis, which was treated by vesicostomy and subsequent permanent suprapubic catheter. This was complicated by hydronephrosis and chronic renal impairment. In early adulthood, he had dental veneers and crowns placed to treat severe enamel defects and caries. As an adult he performed all activities of daily living independently, as well as gym-based exercise with only minimal skin blistering. His supraglottic stenosis was asymptomatic and has been managed conservatively. His most troubling symptom was significant onychodystrophy (Fig. 1) requiring mechanical debridement. He has not had any further symptoms of PLE, but has been unable to gain weight $(55 \mathrm{~kg})$ despite high calorie intake.

Following informed consent from the patient, genetic testing was performed, which revealed a homozygous pathogenic frameshift variant in ITGB4 (c.4631_4632del, p.Leu1544GInfs*27). This variant has been reported once previously in a homozygous state, in a patient with JEB-PA (annotated as 4790 delTC based on an outdated reference transcript). ${ }^{3}$ The features of our patient, his sister and this previously reported case are compared in Table 1.

There have been several reports of ITGB4 variant JEB-PA without PA ${ }^{2,4}$, as in the case of our patient and his sibling; pyloric stenosis is a relatively common neonatal anomaly, but this could also represent a phenotypic extension of JEB-PA. Sibling discordance with regard to PA has been described, suggesting that the PA is due to factors other than genotype. ${ }^{4}$ Presence of PA is not predictive of poor outcome, as mortality seems to be more dependent upon the mucosal manifestations in early life and the effect of the pathogenic variants on level of $\alpha 6 \beta 4$ integrin expression ${ }^{2,5}$

This case demonstrates significant survival disco.rdance despite early concordance of phenotype, between siblings with JEB-PA. Although there are suggestions that patients with nonsense/frameshift mutations have more severe phenotypes than those with missense mutations, this is not an absolute demarcation..$^{2-5}$ We can reasonably expect that both siblings carried the same ITGB4 mutation, therefore negating ITGB4 genotype as a factor in predicting the two different outcomes. It is possible that our proband's background genetic milieu may differ from his sister's despite consanguinity, and there could be as yet unascertained environmental factors that modulate the phenotype. The proband may have simply been fortunate to survive severe illness early in life, while his sister was not as lucky, or perhaps his complications of JEB-PA were managed more effectively in light of his sister's demise. Improvement of JEB-PA manifestations with age is well-documented. ${ }^{2,4}$ It has been postulated that severe PLE in infants with JEB-PA is due to autoimmune mucosal inflammatory responses, perhaps triggered by systemic infections. The proposed mechanism is abnormal antigen exposure secondary to the loss of epithelial barrier function. ${ }^{6,7}$ This susceptibility may improve with age.

This case adds to the current literature by demonstrating phenotypic variability in JEB-PA (including the absence of PA) even in patients with ostensibly the same pathogenic genotype.

\section{References}

1 Fine JD, Bruckner-Tuderman L, Eady RA et al. Inherited epidermolysis bullosa: updated recommendations on diagnosis and classification. J Am Acad Dermatol 2014; 70: 1103-26.

This article is protected by copyright. All rights reserved 
2 Schumann $H$, Kiritsi D, Pigors $M$ et al. Phenotypic spectrum of epidermolysis bullosa associated with $\alpha 6 \beta 4$ integrin mutations. Br J Dermatol 2013; 169: 115-24.

3 Nakano A, Pulkkinen L, Murrell D. Epidermolysis bullosa with congenital pyloric atresia: novel mutations in the beta 4 integrin gene (ITGB4) and genotype/phenotype correlations. Pediatr Res 2001; 49: 618-26

4 Dang $\mathrm{N}$, Klingberg $\mathrm{S}$, Rubin $\mathrm{Al}$ et al. Differential expression of pyloric atresia in junctional epidermolysis bullosa with ITGB4 mutations suggests that pyloric atresia is due to factors other than the mutations and not predictive of a poor outcome: three novel mutations and a review of the literature. Acta Derm Venereol 2008; 88: 438-48.

5 Ashton GH, Sorelli $\mathrm{P}$, Mellerio JE et al. Alpha 6 beta 4 integrin abnormalities in junctional epidermolysis bullosa with pyloric atresia. Br J Dermatol 2001; 144: 408-14.

6 Salvestrini C, McGrath JA, Ozoemena L et al. Desquamative enteropathy and pyloric atresia without skin disease caused by a novel intracellular $\beta 4$ integrin mutation. J Pediatr Gastroenterol Nutr 2008; 47: 585-91.

7 Freeman EB, Koglmeier J, Martinez AE et al. Gastrointestinal complications of epidermolysis bullosa in children. Pediatr Dermatol 2008. 158: 1308-14.

Figure $1(a)$ Fingernail and $(b, c)$ toenail onychodystrophy in the proband with junctional epidermolysis bullosa-pyloric atresia/.

Table 1 Comparison of the phenotypic features of junctional epidermolysis bullosa-pyloric atresia caused by the same pathogenic variant (ITGB4 c.4631_4632del).

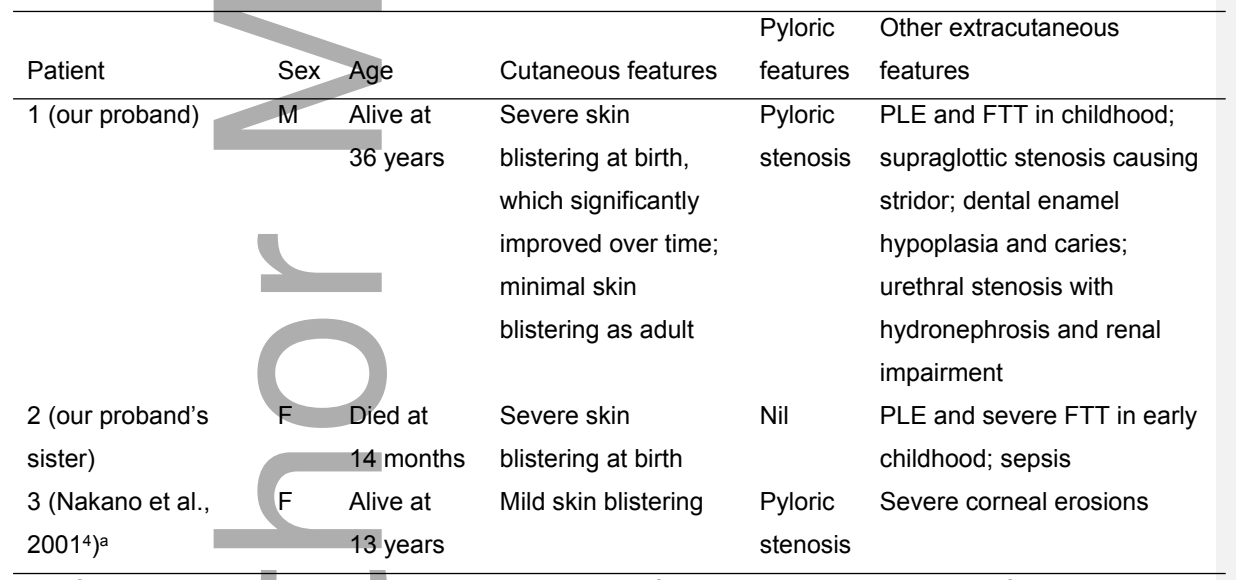

FTT, failure to thrive; PLE, protein-losing enteropathy. ${ }^{\text {IInformation on the clinical state of this individual is }}$ limited to the reference.

\section{CPD questions}

\section{Learning objective}

To demonstrate an understanding of the clinical and genetic features of junctional epidermolysis bullosa-

This article is protected by copyright. All rights reserved 
pyloric atresia.

\section{Question 1}

Which of the following extracutaneous features occur in junctional epidermolysis bullosa-pyloric atresia (JEB-PA) apart from pyloric atresia?

(a) Tracheo-oesophageal fistula.

(b) Pseudosyndactyly and rudimentary ears.

(c) Genitourinary abnormalities and dental caries.

(d) Genitourinary abnormalities and keratoconus.

(e) Jejunal atresia and muscular dystrophy.

\section{Question 2}

Genes coding for which of following proteins are implicated in the pathogenesis of junctional epidermolysis bullosa-pyloric atresia (JEB-PA)?
(a) Integrin $\alpha 6$.
(b) Integrin $\alpha 3$ subunit.
(c) Plectin.
(d) Integrin $\beta 4$.
(e) Laminin-332.

\section{Answer 1}

Which of the following extracutaneous features occur in junctional epidermolysis bullosa-pyloric atresia (JEB-PA) apart from pyloric atresia?

(a) Incorrect. Tracheo-oesophageal fistula is not an extracutaneous manifestation of JEB-PA.

(b) Incorrect. Pseudosyndactyly is not an extracutaneous manifestation of JEB-PA.

(c) Correct. Genitourinary abnormalities and dental caries commonly occur in JEB-PA.

(d) Incorrect. Keratoconus is not an extracutaneous manifestation of JEB-PA.

(e) Incorrect. Neither of these are extracutaneous manifestations of JEB-PA.

\section{Answer 2}

Genes coding for which of following proteins are implicated in the pathogenesis of junctional epidermolysis bullosa-pyloric atresia (JEB-PA)?

(a) Correct. Integrin $\alpha 6$ is encoded by ITGA6 and is implicated in JEB-PA.

(b) Incorrect. ITGA3 encodes Integrin $\alpha 3$ is encoded by and is implicated in JEB with respiratory and renal involvement.

(c) Incorrect. PLEC encodes plectin is encoded by, and is implicated in EB simplex (EBS) with PA, as well as EBS with muscular dystrophy (EBS-MD) and EBS-Ogna.

(d) Correct. ITGB4 encodesIntegrin $\beta 4$ is encoded by and is implicated in JEB-PA.

(e) Incorrect. $\angle A M A 3, \angle A M B 3$ and $L A M C 2$ encode laminin-332 is encoded by, which is implicated in other forms of JEB without PA, namely: JEB generalized-severe, JEB generalized-intermediate, JEB localized, JEB inversa and JEB laryngo-onycho-cutaneous syndrome..

This article is protected by copyright. All rights reserved 

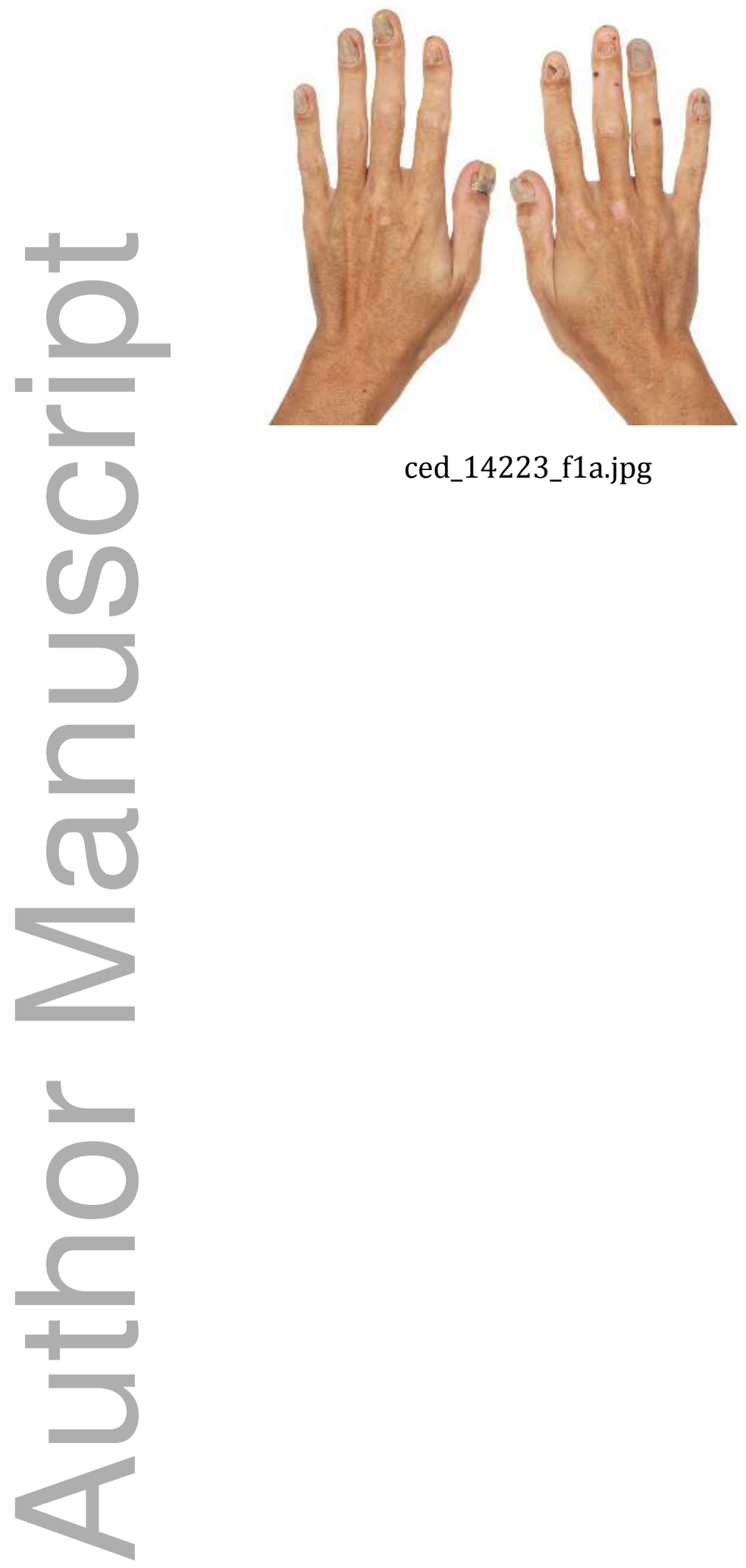

This article is protected by copyright. All rights reserved 

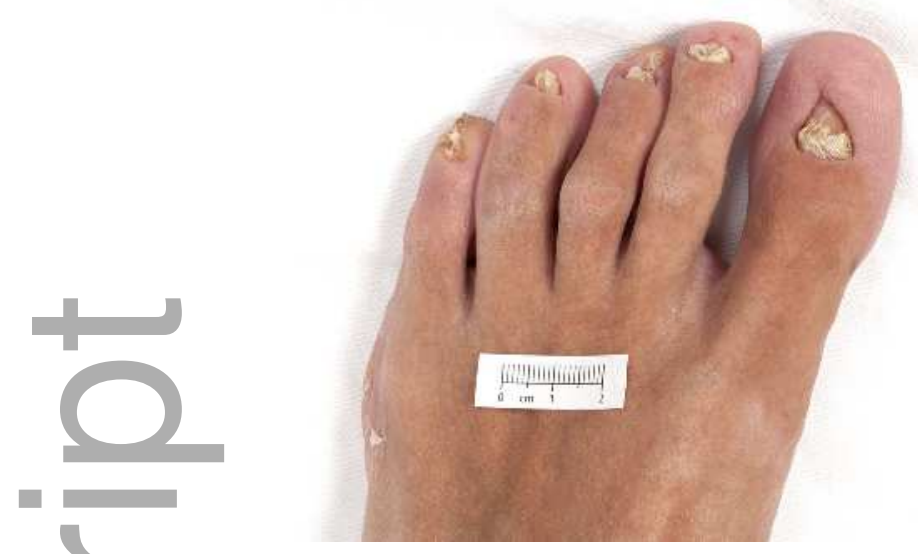

ced_14223_f1b.jpg

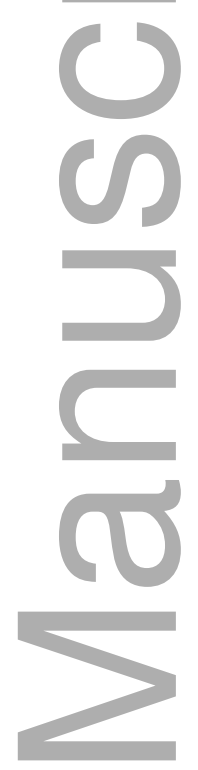

c14223_f1b.jpg

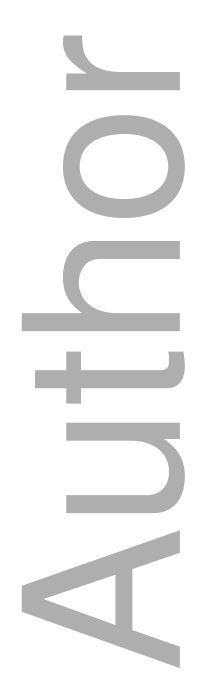

This article is protected by copyright. All rights reserved 

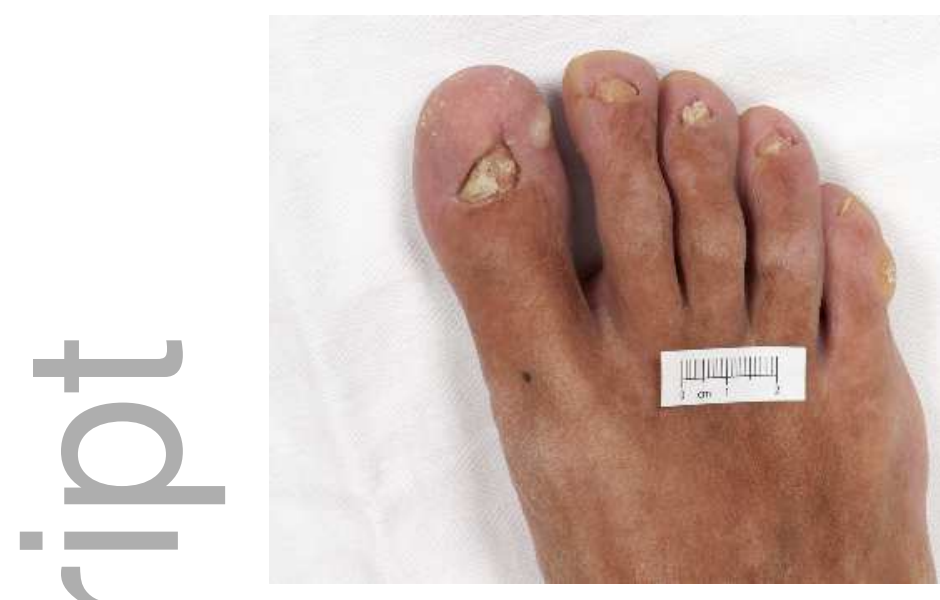

ced_14223_f1c.jpg

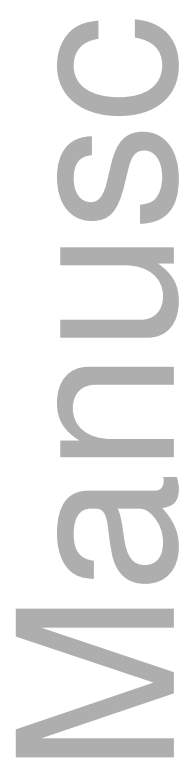

\author{
ced_14223_f1c.jpg
}

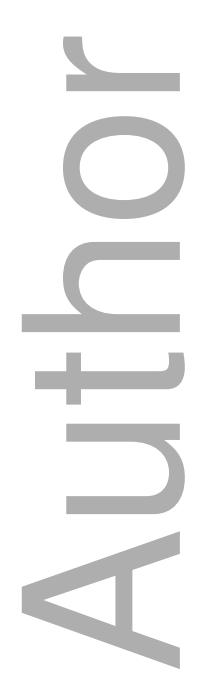

This article is protected by copyright. All rights reserved 


\section{University Library}

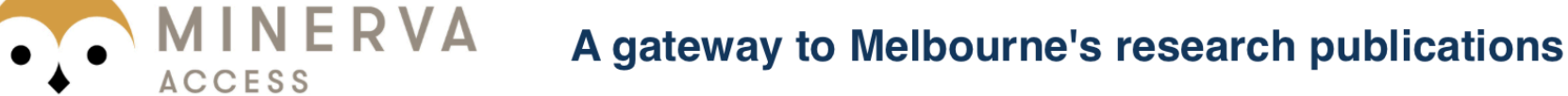

Minerva Access is the Institutional Repository of The University of Melbourne

Author/s:

Verma, KP;Robertson, SJ;Winship, IM

Title:

Phenotypic discordance between siblings with junctional epidermolysis bullosa-pyloric atresia

Date:

2020-05-02

\section{Citation:}

Verma, K. P., Robertson, S. J. \& Winship, I. M. (2020). Phenotypic discordance between siblings with junctional epidermolysis bullosa-pyloric atresia. CLINICAL AND EXPERIMENTAL DERMATOLOGY, 45 (6), pp.793-795. https://doi.org/10.1111/ced.14223.

Persistent Link:

http://hdl.handle.net/11343/275709 